\title{
Research on Modeling and Simulation of controllable weak magnetic field based on Helmholtz coil
}

\author{
Ruifeng Zhang ${ }^{1}$,Wei Chi ${ }^{2}$, Liangan Jin $^{3}$ \\ ${ }^{1}$ Team of Graduate Student, Dalian Naval Academy, Dalian 116018, China \\ ${ }^{2.3}$ Dept. of Navigation, Dalian Naval Academy, Dalian 116018, China
}

Keywords: Controllable weak magnetic field, Helmholtz coils, simulation model.

\begin{abstract}
Controllable weak magnetic field can be generated using circular Helmholtz coils. However, it is complex to calculate the internal magnetic field distribution. This paper mainly expounds the formation mechanism and the simulation method of controllable weak magnetic field. A simulation model is established based on MATLAB. The simulation results demonstrate its effectiveness.
\end{abstract}

\section{Introduction}

In high latitude area, the geomagnetic horizontal component gets so weak. As a result, the existing various magnetic navigation equipment can hardly work. Using artificial controllable magnetic field to neutralize geomagnetic horizontal component is proved to be an effective way to simulate this weak-magnetic environment under laboratory conditions.

Controllable weak magnetic field can be generated using circular Helmholtz coils. Its strength and uniformity can be guaranteed, but the internal magnetic field distribution is complex to calculate. To establish a simulation through theoretical analysis and run Dynamic simulation using MATAB will provide theoretical support for the experimental research.

MATLAB is a scientific computing software launched by Math works for numerical computing and graphics processing. It has comprehensive functions, friendly interface and good opening. In the international academic community, MATLAB has been validated as an accurate, reliable calculation of standard software; in the engineering sector, MATLAB is often used to solve some practical issues and mathematical model.

This paper chooses MATLAB as software tool, using the simulation mode established, to make numerical calculation of the magnetic field strength for any point inside the Helmholtz coil and to picture its distribution curve. The results will provide a more thorough and intuitive understanding of the Helmholtz coils magnetic field.

\section{Generation mechanism of controllable weak magnetic field}

The circular Helmholtz coils are used here to produce controllable magnetic field, which are made of two coaxial circular coils (with same radius). According to the law of electromagnetic induction, current in the coils could produce magnetic field in center area, and its direction accords with right-hand screw rule.

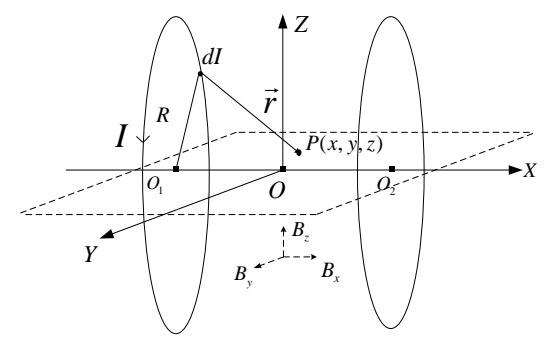

Figure 1. Model for Circular Helmholtz Coils

Figure 1 is a model for circular Helmholtz coils. According to the Biot-Savart law, when the 
current intensity is $\mathrm{I}$, a certain current element IdI on coil ${ }^{O_{1}}$ will produce certain magnetization intensity $d \vec{B}$ at a point $\mathrm{P}$ in space:

$$
d \vec{B}=\frac{\mu_{0}}{4 \pi} \cdot \frac{I d \vec{I} \times \vec{e}_{r}}{r^{2}}=\frac{\mu_{0} I}{4 \pi} \cdot \frac{d \vec{I} \times \vec{r}}{r^{3}}
$$

In Formula 1, vector $\vec{r}$ means the radius vector for IdI to P. $\mu_{0}$ is the permeability of vacuum, which is $4 \pi \times 10^{-7} \mathrm{~T} \cdot \mathrm{m} / \mathrm{A}$.

The $d \vec{B}$ integral of $d I$ can obtain the magnetic field intensity by circular current at any point in space. A simulation model can be established according to Formal 1 using the coordinate system in Figure 1.

Divide the circumference of $\mathrm{O} 1$ into $\mathrm{K}$ segments. When $\mathrm{K}$ is big enough, each segment can be seem approximately as a current element. The starting point and end point of segment k corresponds an angle of $\theta_{k}$ and $\theta_{k+1}$.

Calculate the magnetic field strength of each segment on Point $\mathrm{P}$, and then add all $\mathrm{K}$ segments up to obtain the total magnetic induction intensity of Coil O1. Due to the symmetry, that of Coil O2 can be obtained from the translation of $\mathrm{O} 1$. The vector sum of the two coils' magnetic intensity makes the total magnetic induction intensity of Helmholtz Coils on Point P.

To Segment k on Coil O1. Using the coordinate system above, the coordinate of its start point and end point is: $\left(-R / 2, R \cos \theta_{k}, R \sin \theta_{k}\right)$ and $\left(-R / 2, R \cos \theta_{k+1}, R \sin \theta_{k+1}\right)$.

The midpoint coordinate is:

$$
\left[-\frac{R}{2}, \frac{R\left(\cos \theta_{k+1}+\cos \theta_{k}\right)}{2}, \frac{R\left(\sin \theta_{k+1}+\sin \theta_{k}\right)}{2}\right] \text {. }
$$

Use this midpoint to approximately replace the spatial location of that current element, and also to be the start point of its radius vector. To $\mathrm{P}(\mathrm{x}, \mathrm{y}, \mathrm{z})$, radius vector $\vec{r}$ from the midpoint of current element $d \vec{l}$ to it is described as Formula 2:

$$
\left\{\begin{array}{c}
\overrightarrow{d l}=\left(0, R \cos \theta_{k+1}-R \cos \theta_{k}, R \sin \theta_{k+1}-R \sin \theta_{k}\right) \\
\vec{r}=\left[x+\frac{R}{2}, y-\frac{R\left(\cos \theta_{k+1}+\cos \theta_{k}\right)}{2},\right. \\
\left.z-\frac{R\left(\sin \theta_{k+1}^{2}+\sin \theta_{k}\right)}{2}\right]
\end{array}\right.
$$

The length of $\vec{r}$ can be calculated through Formula 3:

$$
r=\sqrt{\begin{array}{r}
\left(x+\frac{R}{2}\right)^{2}+\left(y-\frac{R\left(\cos \theta_{k+1}+\cos \theta_{k}\right)}{2}\right)^{2} \\
+\left(z-\frac{R\left(\sin \theta_{k+1}^{2}+\sin \theta_{k}\right)}{2}\right)^{2}
\end{array}}
$$

Take Formula 2 and 3 into Formula 1 to obtain the analytic formula of the magnetic field intensity $d \vec{B}$ on Point P from Segment k. Its three component along the coordinate axis is as Formula 4. (Next Page)

Through the summation operation to $\mathrm{k}$ in the Formula 4, we can get the total magnetic field intensity on Point P from Coil O1.

Due to the space symmetry, the magnetic induction intensity of Coil O2 is Equivalent to that of Coil $\mathrm{O} 1$ with a translation alongside the positive direction of axis $\mathrm{X}$ at a distance of $\mathrm{x}=\mathrm{R}$. Add up the magnetic intensity of two coils to get the total magnetic field strength on Point $P$ from the Helmholtz Coils.

Through looping summary of each component of the coordinate of $\mathrm{P}(\mathrm{x}, \mathrm{y}, \mathrm{z})$, we can study the 
magnetic distribution on any point inside the Helmholtz Coils.

$$
\left\{\begin{array}{l}
d \vec{B}_{x}=\frac{\mu_{0} I R}{4 \pi} \cdot \frac{\left(z-\frac{R\left(\sin \theta_{k+1}+\sin \theta_{k}\right)}{2}\right) \cdot\left(\cos \theta_{k+1}-\cos \theta_{k}\right)-\left(y-\frac{R\left(\cos \theta_{k+1}+\cos \theta_{k}\right)}{2}\right) \cdot\left(\sin \theta_{k+1}-\sin \theta_{k}\right)}{\sqrt{\left(x+\frac{R}{2}\right)^{2}+\left(y-\frac{R\left(\cos \theta_{k+1}+\cos \theta_{k}\right)}{2}\right)^{2}+\left(z-\frac{R\left(\sin \theta_{k+1}+\sin \theta_{k}\right)}{2}\right)^{2}}} \\
d \vec{B}_{y}=\frac{\mu_{0} I R}{4 \pi} \cdot \frac{\left(\sin \theta_{k+1}-\sin \theta_{k}\right)\left(x+\frac{R}{2}\right)}{\sqrt{\left(x+\frac{R}{2}\right)^{2}+\left(y-\frac{R\left(\cos \theta_{k+1}+\cos \theta_{k}\right)}{2}\right)^{2}+\left(z-\frac{R\left(\sin \theta_{k+1}+\sin \theta_{k}\right)}{2}\right)^{2}}} \\
d \vec{B}_{z}=\frac{\mu_{0} I R}{4 \pi} \cdot \frac{\left(\cos \theta_{k+1}-\cos \theta_{k}\right)\left(x+\frac{R}{2}\right)}{{\sqrt{\left(x+\frac{R}{2}\right)^{2}+\left(y-\frac{R\left(\cos \theta_{k+1}+\cos \theta_{k}\right)}{2}\right)^{2}+\left(z-\frac{R\left(\sin \theta_{k+1}+\sin \theta_{k}\right)}{2}\right)^{2}}}^{3}}
\end{array}\right.
$$

\section{Simulation Results}

For the analysis of the uniformity, the magnetic field uniformity index is defined as:

$$
\xi=\frac{\left|B_{x}-B_{O}\right|}{B_{O}} \times 100 \%
$$

The simulation model is deduced from formula 1, and it can be used to investigate the magnetic field strength and uniformity at any point in center space.

Simulation parameters are as follows: Radius of coils $R=0.5 \mathrm{~m}$, the number of turns $N=20$, and the excitation current $I=1 \mathrm{~A}$.

The simulation results are as Fig. 2.

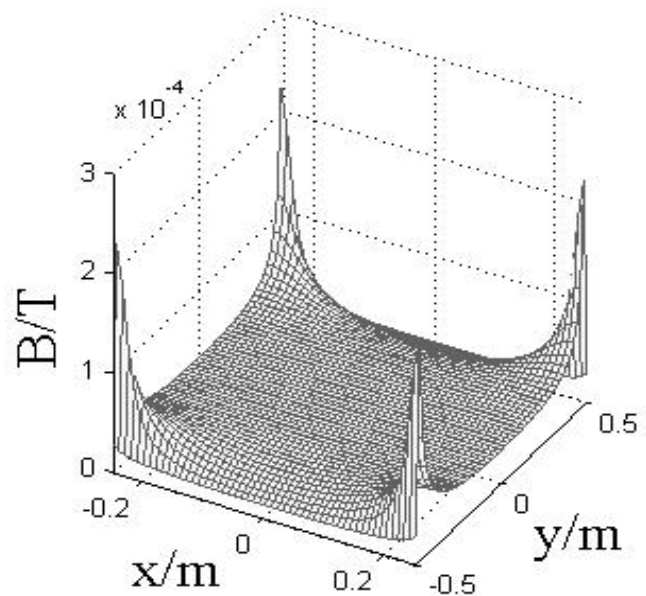

(a) $\mathrm{Bx}$

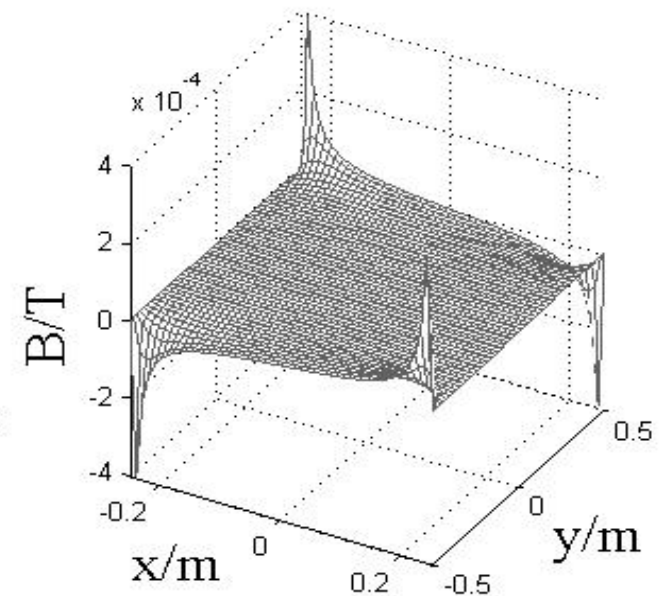

(b) By

Figure 2. Magnetic field distribution on XOY plane

Figure 2 shows the magnetic field distribution on the center plane XOY by the simulation model. In Fig. 2(a), there is a large flat region which means the existence of a large uniform magnetic field in center area. In Fig. 2(b), the vertical component stays 0 in a large range, which means that the resultant vector is alongside the axis direction.

Because the magnetic strength on the center axis of the coil have more clear and explicit expressions, we can choose these points as samples to test the simulation model. The magnetic field intensity at a point $(\mathrm{x}, 0,0)$ on the center axis is:

$$
B_{x}=\frac{\mu_{0} N I \cdot R^{2}}{2\left[R^{2}+\left(\frac{R}{2}+x\right)^{2}\right]^{3 / 2}}+\frac{\mu_{0} N I \cdot R^{2}}{2\left[R^{2}+\left(\frac{R}{2}-x\right)^{2}\right]^{3 / 2}}
$$

Choose some points on center axis and compare the theoretical value (TV) and the simulation value (SV). The results are as Tab.1: 
Table.1 Results Comparison on center axis

\begin{tabular}{ccc}
\hline$X / \mathrm{cm}$ & $T V / 10^{-5} \mathrm{~T}$ & $S V / 10^{-5} \mathrm{~T}$ \\
\hline 0 & 3.5967 & 3.5967 \\
10 & 3.5904 & 3.5904 \\
25 & 3.4018 & 3.402 \\
\hline
\end{tabular}

The data comparison results in Tab.1 shows that, the simulation model can effectively simulate the magnetic field distribution of Helmholtz coils.

Take the center point $\mathrm{O}$ as reference point, and set $\xi<2 \%$. Use our simulation model to calculate the magnetic strength on different points of different planes and compare the simulation values to the reference value.Fig.3 shows the range of uniform area on different plane and its variation trend.

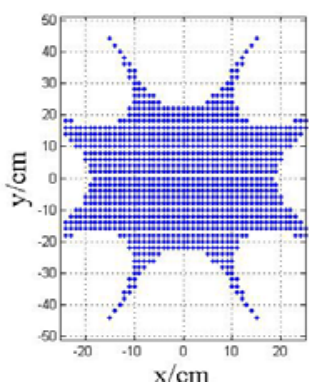

(a) $\mathrm{Z}=0$

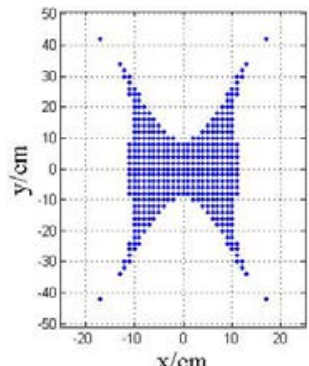

(c) $\mathrm{Z}=20 \mathrm{~cm}$

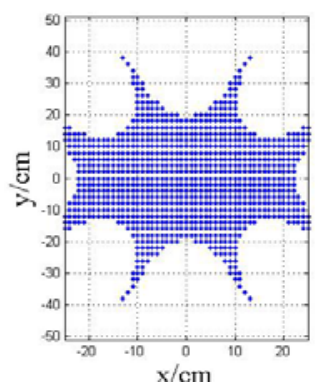

(b) $\mathrm{Z}=10 \mathrm{~cm}$

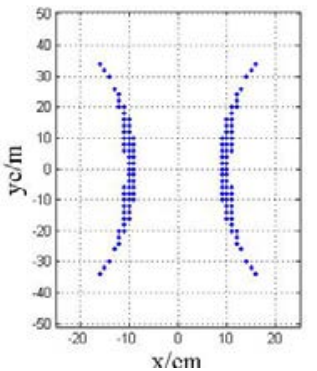

(d) $\mathrm{Z}=30 \mathrm{~cm}$

Figure 3. Range of uniform area on different planes

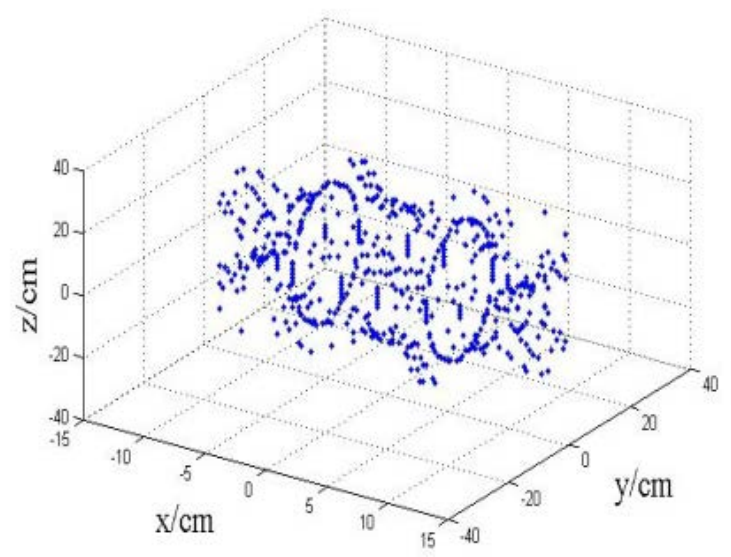

Figure 4. Space boundary of uniform area

Fig.3 shows the comparison of the uniform magnetic field area on different simulation planes. The results show that, the magnetic field can be uniform within a large range in center space. The range decreases to both sides along the $\mathrm{Z}$ axis. Fig. 4 shows the space boundary of uniform area. We can see from the figure that, the space boundary is overall approximately cylindrical, while the 
variation trend is similar to Fig.3.

\section{Conclusion}

MATLAB is a practical software with the functions of numerical calculation and dynamic simulation. With an effective simulation model, it can provide accurate data and Intuitive graphics for further analysis and experimental research.

The simulation results show that, the simulation model established in this paper has certain reliability. It can effectively describe the magnetic distribution of the Helmholtz Coils and be used to simulate the controllable weak magnetic environment.

\section{Reference}

[1] Liu Kun, Zhang Songyong. Analysis on magnetic field homogeneity of magnetic system based on square Helmholtz coils [J]. Modern Electronic Technique, 2012, 35(7):190-193.

[2] Si Wenjian, Zhou Nan. Dynamic Simulation of Uniform Distribution of the Telmholtz Coil Magnetic Field on MATLAB [J]. Journal of Xuchang University, 2005, 29(5):72-74.

[3] Tan Xi, The Magnetic Field Uniformity of Square Helmholtz Coils [J].Optical Instrument, 2012, 34(1):39-44.

[4] Yang Xiaodong, Study on Geomagnetic Navigation Technology in High Latitude Area [J], Ship \& Ocean Engineering, 2010, 39(5): 128-133.

[5] Zhang Chang-xin, Study on Magnetic Field of a Circular Current and Helmholtz Coils [J]. Journal of Anhui Normal University (Natrual Science), 2004. 27(1): 41-45. 